\title{
Revised Consistency Conditions for PET Data
}

\author{
J. L. Herraiz ${ }^{1}$, S. España ${ }^{1}$, E. Vicente ${ }^{1}$, E. Herranz ${ }^{1}$, J. J. Vaquero ${ }^{2}$, M. Desco ${ }^{2}$, J. M. Udías ${ }^{1}$ \\ ${ }^{1}$ Grupo de Física Nuclear, Universidad Complutense, Madrid, Spain \\ ${ }^{2}$ Unidad de Medicina y Cirugía Experimental, Hospital GU “Gregorio Marañón”, Madrid, Spain
}

\begin{abstract}
Tomographic Data Consistency Conditions (TDCC) are frequently employed to improve the quality of PET data. However, most of these consistency conditions were derived from X-ray computerized tomography (CT) and their validity for other imaging modalities has not been well established. For instance, it is well known from (X-ray) CT data that the sum of the projection data from one view of the parallel-beam projections is a constant independent of the view-angle. This consistency condition is based on well-known mathematical properties of the Radon transform and yields good results when employed in noise removal or sinogram restoration. But this consistency condition assumes that emission and detection of radiation occur within a thin (ideally with zero width) line-ofresponse (LOR), with a flat probability distribution of the detection (in PET) or absorption (X-ray) along such LOR. This assumption, being valid for $\mathrm{CT}$, is not realistic for PET acquisitions. Thus, TDCC for PET should be revised in order to check their validity with more realistic detection models.

In this work we review the main differences between PET and CT data and study whether these consistency conditions should be modified in order to take into account the dependence of the probabilities on the distance to the center of the line-of-response. Results from simulations are also presented to illustrate the importance of these effects. They indicate that some consistency conditions can be violated at the $10 \%$ level.
\end{abstract}

\section{INTRODUCTION}

$I^{\mathrm{T}}$ is well known from (X-ray) CT data that the sum of the projection data from one view of the parallel-beam projections is a constant independent of the view-angle. This consistency condition of tomographic data is based on wellknown mathematical properties of the Radon transform [1] and yields good results when employed in noise removal [2], sinogram restoration [3, 4] and attenuation correction [5].

Nevertheless, these kind of mathematical properties assume that emission and detection of radiation occur within a thin (ideally with zero width) line-of-response (LOR), with a flat distribution of the probability along the LOR. This assumption, being valid for CT, is not completely realistic for PET and SPECT acquisitions [6-9]. In SPECT some efforts have been devoted to take into account this spatially variant detector response [9], but the impact of it on TDCC has not been fully addressed. The differences between PET and CT

Manuscript received November 23, 2007.

J. L. Herraiz e-mail: joaquin@nuclear.fis.ucm.es.

S. España e-mail: samuel@nuclear.fis.ucm.es,

E.Vicente email: evicente@nuclear.fis.ucm.es,

E. Herrnaz e-mail: eherranz@nuclear.fis.ucm.es,

J.J.Vaquero email: juanjo@mce.hggm.es,

M. Desco e-mail: desco@mce.hggm.es;

J.M.Udias e-mail: jose@nuc2.fis.ucm.es sinograms have significant implications for TDCC, making them only approximately valid for PET. In extreme cases, the use of TDCC may even result in wrong conclusions and methods.

In this work, we have studied with simulations and real acquisitions to which extent realistic sinogram models deviate from the ideal case. Attenuation of the gamma rays and scatter has not been considered as we have used point sources in air. Nevertheless, the results of this paper can be directly extended to real PET acquisitions once corrected for attenuation.

\section{TOMOGRAPHIC DATA CONSISTENCY CONDITIONS IN PET}

\section{A. Ideal Sinograms}

Using a discretized representation for the field of view (FOV) of the scanner, each 2-Dimensional sinogram of the acquired data with radial and angular coordinates $(\xi, \theta)$ can be decomposed as a sum of contributions from different source points. Ideally, it can be described using zero-width Dirac delta functions:

$$
p(\xi, \theta)=\sum_{i}^{N} \omega_{i} \cdot \delta\left(\xi-r_{i \theta}\right)
$$

where $r_{i, \theta} \equiv R_{i} \cdot \sin \left(\theta-\phi_{i}\right)$, being $R_{i}$ and $\phi_{i}$ the polar coordinates of the source point $\mathrm{i}$ [Fig. 1]. N represents the total number of points in the FOV and the weight $\omega_{i}$ is proportional to the intensity of the activity at point $i$.
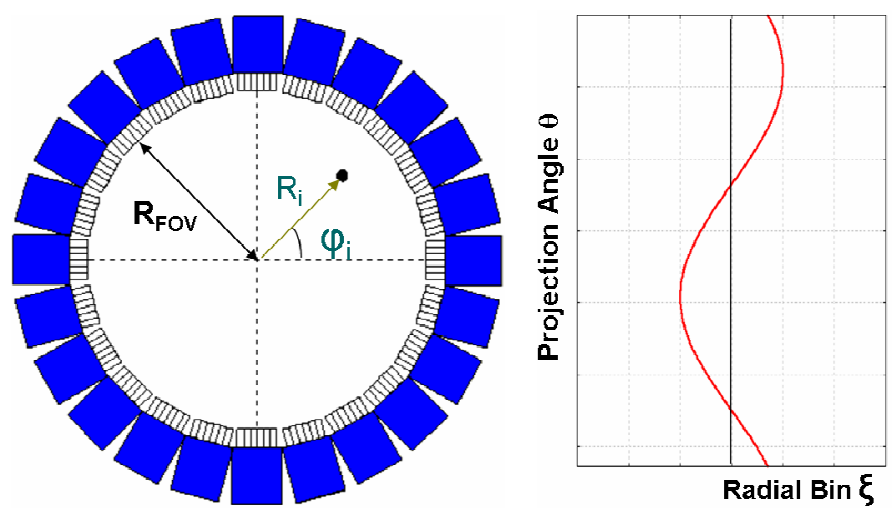

Fig. 1. (Left) Coordinates of a point source inside a PET scanner. (Right) Ideal Sinogram of the projection data for this point source.

Several properties of the sinogram can be easily derived from (1). For example, the sum of all radial bins inside the Field-of-View of the scanner (FOV) corresponding to a fixed angle $\theta=\theta_{0}$ is a view-independent constant: 


$$
\left.\sum_{\xi=-R_{F O V}}^{+R_{F O V}} p(\xi, \theta)\right|_{\theta=\theta_{0}}=\sum_{i}^{N} \omega_{i}=\text { cons tan } t
$$

$\mathrm{R}_{\mathrm{FOV}}$ represents the radius of the FOV of the scanner. This result is the zero-order version of the often called HelgasonLudwig conditions. Further details can be found in reference [1]. This result is closely related to the Bow-Tie principle commonly applied in FORE [10]. $\mathrm{Q}_{0}$ (theta) $=$ constant is equivalent to say that in a 2D Fourier Transform of the sinogram all elements with radial frequency zero should be zero except $m=0$.

In general, these conditions state that the moment $Q_{n}$ $(n=0,1,2 \ldots)$ of the sinogram defined as:

$$
Q_{n}(\theta) \equiv \sum_{\xi=-R_{F O V}}^{+R_{F O V}} \xi^{n} \cdot p(\xi, \theta)
$$

for an ideal sinogram as described by (1) corresponds to:

$$
Q_{n}(\theta)=\sum_{i}^{N} \omega_{i} \cdot r_{i}^{n}=\sum_{i}^{N} \omega_{i} \cdot R_{i}^{n} \cdot \sin ^{n}\left(\theta-\phi_{i}\right)
$$

Using trigonometric relations for the powers of the sine, the moment $\mathrm{Q}_{\mathrm{n}}$ can be expanded out as homogeneous polynomials

\begin{tabular}{|c|c|c|}
\hline ORDER & MEANING & GRAPHIC PLOT \\
\hline$Q_{0}(\theta)$ & The sum of projections & 1.2 \\
\hline $\mathrm{N}=0$ & angles & , \\
\hline$Q_{1}(\theta)$ & $\begin{array}{l}\text { The projections of the } \\
\text { center of mass of the } \\
\text { object form a sine }\end{array}$ & \\
\hline $\mathrm{N}=1$ & $\begin{array}{l}\text { wave with period } 1 \text { in } \\
\text { the sinogram }\end{array}$ & $-\infty$, \\
\hline$Q_{2}(\theta)$ & Sine wave with period & \\
\hline $\mathrm{N}=2$ & 2 in the sinogram. & \\
\hline
\end{tabular}
of order $\mathrm{n}$ in $\sin (\theta)$ and $\cos (\theta)$. This result is explained in Table 1 for $\mathrm{n}=0,1,2$.

Table 1 - Explanation of the meaning of the momentum of order $n=0,1,2$ of the sinogram.

Using the Fourier Transform of (4) respect to the polar angle $\theta$, this TDCC can be summarized as follows: "The Fourier transform of the $\mathrm{Q}_{\mathrm{n}}$ moment of a sinogram (properly corrected by attenuation), should have equals to zero all coefficients greater than $\mathrm{n}$ ".

\section{B. Realistic PET sinograms with symmetric blurring}

In PET, if we take into account the finite size of the crystals and the physical processes involved in the emission and detection of radiation, sinograms are more accurately described by gaussian functions instead of (1) that uses zerowidth Dirac delta functions:

$$
p(\xi, \theta)=\sum_{i}^{N} \frac{\omega_{i}}{\sqrt{2 \pi \cdot \sigma}} \cdot \exp \left(-\frac{\left(\xi-r_{i \theta}\right)^{2}}{2 \sigma^{2}}\right)
$$

The value of $\sigma$ is related to all relevant blurring effects involved in PET such as the finite detector size, positron range, non-collinearity and inter-crystal scatter of detected photons. It is well known [11], that in commonly used ringbased PET system, $\sigma$ is not constant and it becomes wider as the radial distance increase because of the gamma-ray penetration of the detectors. This effect is called Depth-ofInteraction (DOI).

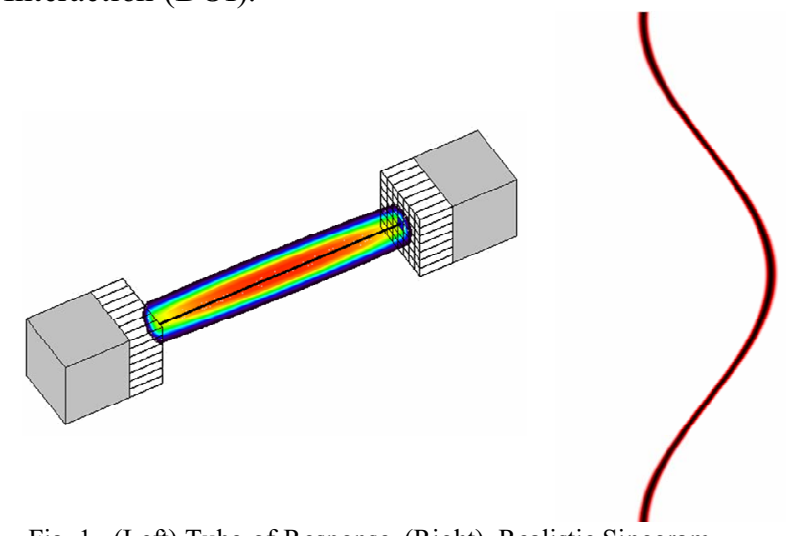

Fig. 1. (Left) Tube-of-Response. (Right) Realistic Sinogram

Due to geometrical considerations, $\sigma$ depends also on the distance of the source to the detectors $t_{i, \theta} \equiv R_{i} \cdot \operatorname{Cos}\left(\theta-\phi_{i}\right)$. This is commonly known as tangential blurring [11] .

As a result of all these effects, $\sigma$ is not a constant but a function of $\mathrm{r}_{\mathrm{i}}$ and $t_{i, \theta}$ :

$$
\sigma=\sigma\left(r_{i \theta}, t_{i, \theta}\right)
$$

In a previous work [6], we focused on the impact of this blurring in the resolution of reconstructed images and the quality of the data. Here we focus in the effect on the TDCC.

\section{Realistic PET sinograms with asymmetric blurring}

In order to make the sinogram model even more realistic, the asymmetric in the blurring caused by the DOI can be taken into account using two gaussians for describing the blurring [11].

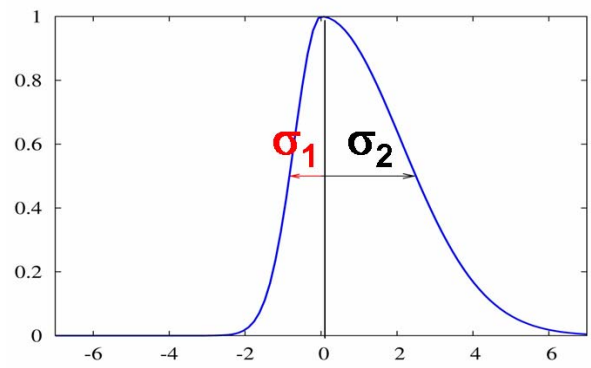

Fig. 2. Radial PSF with asymmetric blurring

Therefore, the model of the sinogram can be expressed in a more general form as: 


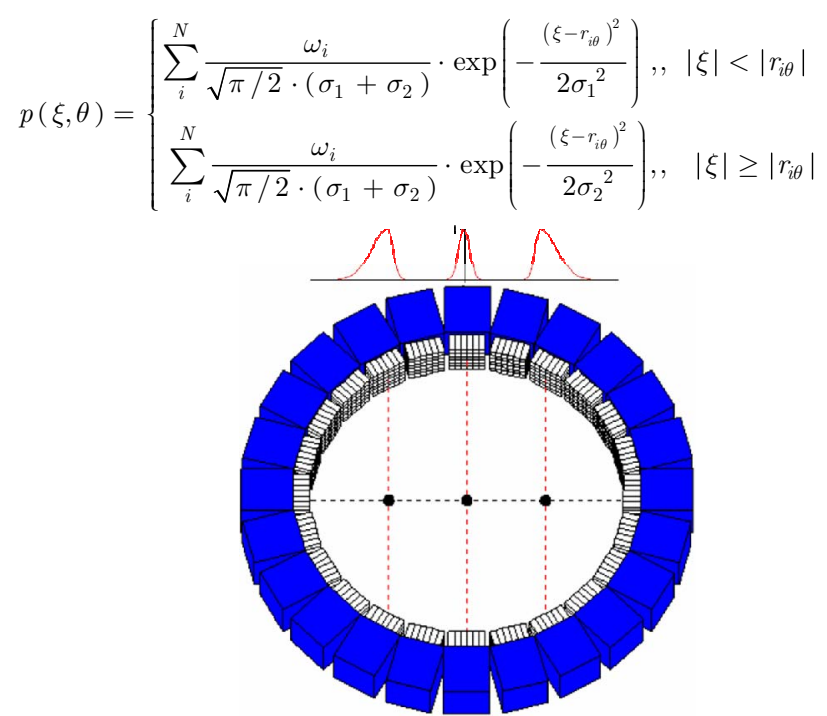

Fig. 2. Non-symmetric blurring caused by the DOI in a ring-based PET scanner for 3 LORs with different radial distance.

This asymmetry is not constant and depends on the radial distance $\mathrm{r}$. The asymmetry of the blurring is defined as $\Delta \sigma \equiv \sigma_{1}-\sigma_{2}$. To show the impact of this asymmetry on the TDCC we will assume a dependency of $\Delta \sigma(\xi)$ as:

$$
\Delta \sigma(\xi)=\delta_{\Delta \sigma} \cdot\left(\frac{\xi}{R_{F O V}}\right)^{2}
$$

$\delta_{\Delta \sigma}$ represents the higher asymmetry of the blurring observed (at the edge of the FOV). Of course the actual expression would depend on the specific parameters of the scanner (crystal pitch, length of the crystals), but we want to make an estimation of the impact of this $\Delta \sigma$ on the TDCC.

We can evaluate the momentum $\mathrm{Q}_{\mathrm{n}}$ of the sinogram substituting the more realistic expression (7) for the sinogram in (3). For simplicity in the rest of this paper we will focus in the first order moments $(n=0,1,2)$ as they are the most commonly used in the references. In this case we obtain:

\begin{tabular}{|c|c|c|}
\hline ORDER & IDEAL & REALISTIC \\
\hline$Q_{0}(\theta)$ & $\sum_{i}^{N} \omega_{i}=K$ & $\sum_{i}^{N} \omega_{i}=K$ \\
\hline$Q_{1}(\theta)$ & $\sum_{i}^{N} \omega_{i} \cdot r_{i \theta}$ & $\sum_{i}^{N} \omega_{i} \cdot\left[r_{i \theta}+\sqrt{\frac{2}{\pi}} \cdot \Delta \sigma\right]$ \\
\hline$Q_{2}(\theta)$ & $\sum_{i}^{N} \omega_{i} \cdot r^{2}{ }_{i \theta}$ & $\sum_{i}^{N} \omega_{i} \cdot\left[r_{i \theta}{ }^{2}+\Delta \sigma^{2}+\sigma_{1} \cdot \sigma_{2}\right.$ \\
& & $\left.+2 \cdot \sqrt{\frac{2}{\pi}} \cdot \Delta \sigma \cdot r_{i \theta}\right]$ \\
\hline
\end{tabular}

Table 2 - Comparison of first order sinogram moments for ideal (no blurring) and realistic (considering the asymmetric blurring) projection data. reason is clear: due to the non-symmetric distribution of the detection probability respect to the center of the LOR, the sinusoidal curve described by the center of mass of the object will be extended or shifted towards higher radial distances from the center. Therefore Q1 will not be a perfect sine function.

If we compare the first order momentum of the sinogram of one point source using the ideal and the realistic model, we obtain:

$$
\begin{array}{r}
\frac{\left.Q_{1}(\theta)\right|_{R E A L}}{\left.Q_{1}(\theta)\right|_{I D E A L}}=\frac{r_{i \theta}+\sqrt{2 / \pi} \cdot \Delta \sigma}{r_{i \theta}}=1+\sqrt{\frac{2}{\pi}} \cdot \frac{\Delta \sigma}{r_{i \theta}} \\
=1+\left(\sqrt{\frac{2}{\pi}} \cdot \frac{\delta_{\Delta \sigma}}{R_{F O V}}\right) \cdot\left(\frac{R_{i}}{R_{F O V}}\right) \operatorname{Sin}\left(\theta-\phi_{i}\right)
\end{array}
$$

For typical values from real PET scanners, if the point source is placed at the edge of the FOV, we can estimate this ratio to be about $\approx\left(1+0.05 \cdot \operatorname{Sin}\left(\theta-\phi_{i}\right)\right)$.

For higher order momentum, the discrepancy between both models increases, as the dependency of the momentum with the blurring and its asymmetry becomes more important.

These results imply that TDCC for PET as described in [12, 13] are only approximately valid. This may have important implications depending on the method where they the TDCC are used. For example, automatic attenuation correction methods $[13,14]$ should pay attention to the particular value of the blurring of the PET scanner because it may invalidate the correction method if the stronger emission sources are not centered as it often occurs with heart or kidney studies.

In the rest of this paper we will verify these results with simulations and real data.

\section{SIMULATIONS}

These results were first tested with some simulations. We wanted to measure in realistic PET data to which extent when dealing with a non-centered source, the TDCC holds true.

We used a Monte Carlo code (PeneloPET) [15] that includes all relevant physical effects, such as positron range, photon non-collinearity, pile-up and inter-crystal scatter.

The geometry of scanner used for the simulations [with long crystals with a small width] was chosen to clearly show the asymmetry in the PSF caused by the DOI. Simulations of point sources placed at different positions inside the FOV were done, obtaining the blurred and asymmetric radial profiles of the probability distribution for three different LORs.

In a second step, a capillar at $3.0 \mathrm{~mm}$ off-center was simulated and the moments of the sinogram and its Fourier Transform were calculated.
This result implies that all moments higher than the zeroorder one should take into account the asymmetry in the sinogram blurring. In the case of the first-order momentum the 

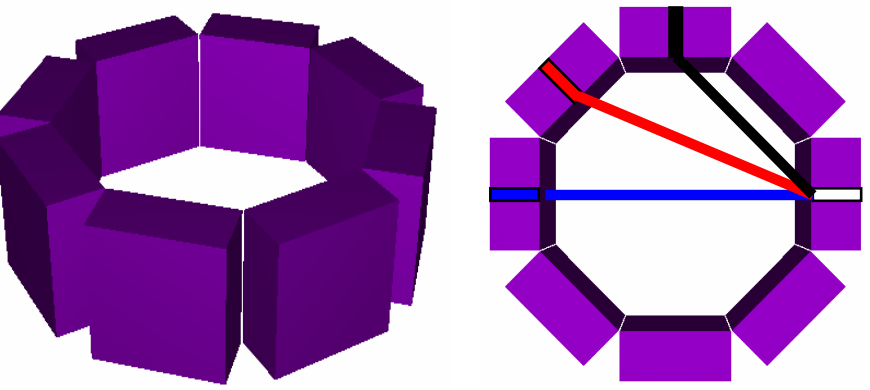

Fig. 3. (Left) Detectors employed in simulations with PeneloPET. (Right) LORs chosen to obtain the radial profile in the probabilities.

\begin{tabular}{|c|c|}
\hline Scintillators & LSO \\
\hline Crystal pitch & $1.0 \mathrm{~mm}$ \\
\hline Crystal length & $20 \mathrm{~mm}$ \\
\hline Scanner size & $80 \mathrm{~mm} \varnothing$ \\
\hline Radioisotope & $18 \mathrm{~F}$ \\
\hline Source size & $5 \mathrm{~mm} \varnothing$ \\
\hline Mean counts per bin & 100 \\
\hline
\end{tabular}

Table 3 - Comparison of first order sinogram moments for ideal (no blurring) and realistic (considering the asymmetric blurring) projection data.

\section{REAL DATA}

Real data from the GE eXplore Vista small animal PET scanner were acquired to test the limits of TDCC on real data. A $1.2 \mathrm{~mm} \varnothing$ capillar filled with $122 \mu \mathrm{Ci}$ of $18 \mathrm{~F}$ were acquired in different positions ( 5 ' per location) inside the FOV and the sinograms were fitted to the Realistic Model of the Sinogram (7) using a genetic algorithm. After that, the Fourier Transform respect to the projection angle of the fit was calculated, obtaining its Fourier Coefficients.

This fitting procedure was necessary because of the existence of gaps in the sinograms. These gaps occur always in this kind of block-based detector scanners, and some procedures like iterative restoration [16], or imposing some conditions [17] on the unknown data allows restoring the sinogram. In this work we didn't want to use none of these methods in order to avoid possible distortions on the results.

\section{RESULTS}

Figure 4 shows the results from the simulation of the asymmetric blurring caused by the DOI. Each color line corresponds to a LOR represented in figure 3.

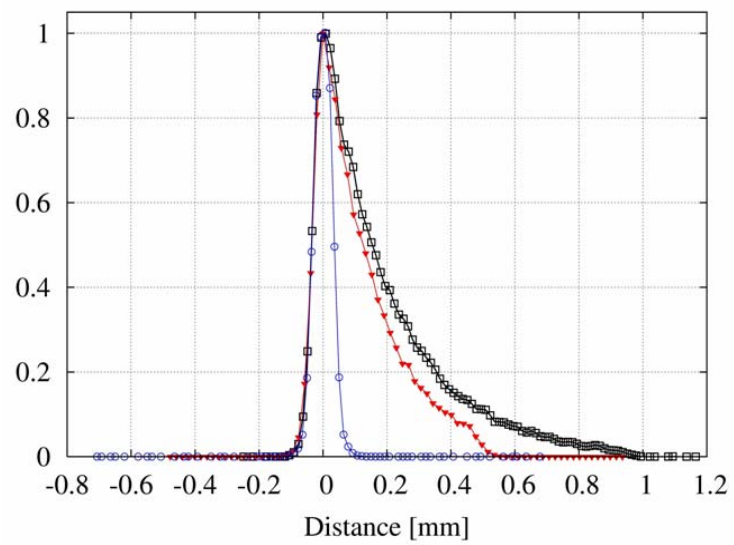

Fig. 4. Radial Point-Spread-Function for 3 different LORs.

Based on these results, we obtained the second-order moment of the ideal sinogram and the simulated one, as shown in figure 5. In order to better quantify these differences, the Fourier Transform of these sine curves is shown in Table 4. It can be seen that there are non negligible differences in Fourier Coefficients respect to the ideal case.

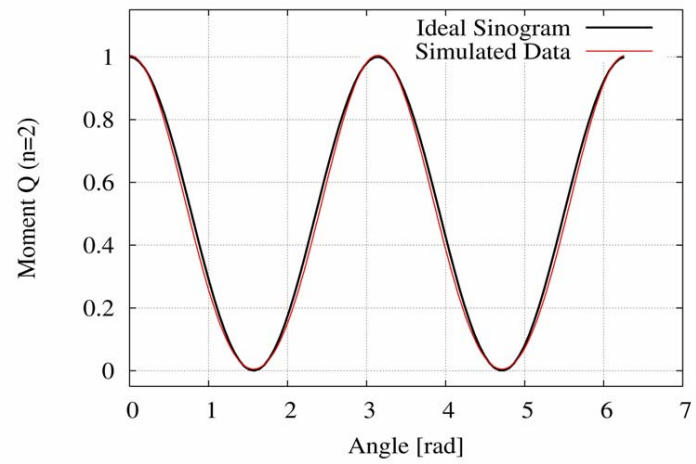

Fig. 5. Second order moment of the simulated sinogram (with realistic blurring) and the ideal sinogram (without blurring).

\begin{tabular}{|c|c|c|}
\hline $\begin{array}{c}\text { Fourier } \\
\text { Coefficient }\end{array}$ & Ideal & Simulation \\
\hline C0 & 0.5 & 0.47 \\
\hline C1 & 0 & $<10^{-4}$ \\
\hline C2 & 0.5 & 0.5 \\
\hline C3 & 0 & $<10^{-4}$ \\
\hline C4 & 0 & 0.03 \\
\hline
\end{tabular}

Table 4 - Second order moment of the simulated sinogram (with realistic blurring) and the ideal sinogram (without blurring).

One of the direct sinograms acquired from a point source in the eXplore Vista scanner is shown in figure 6. 

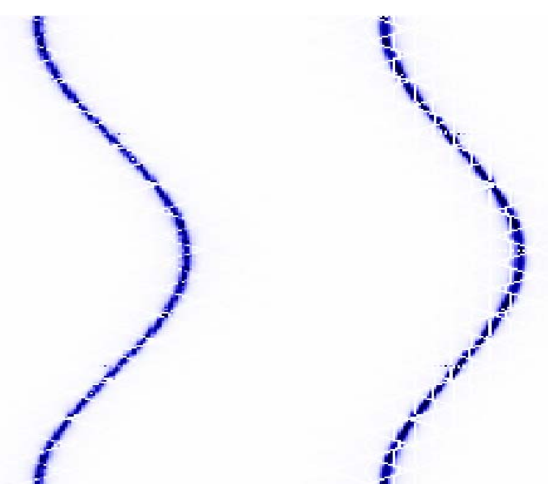

Fig. 6. Acquired sinogram with phoswich (Left) and without phoswich information (Right)

\begin{tabular}{|c|c|c|c|}
\hline $\begin{array}{c}\text { Fourier } \\
\text { Coefficient }\end{array}$ & Ideal & Data & $\begin{array}{c}\text { Data w/o DOI } \\
\text { correction }\end{array}$ \\
\hline C0 & 0.5 & 0.496 & 0.48 \\
\hline C1 & 0 & $<10^{-4}$ & $<10^{-4}$ \\
\hline C2 & 0.5 & 0.5 & 0.5 \\
\hline C3 & 0 & $<10^{-4}$ & $<10^{-4}$ \\
\hline C4 & 0 & 0.004 & 0.02 \\
\hline
\end{tabular}

Table 5 - Second order moment of the simulated sinogram (with realistic blurring) and the ideal sinogram (without blurring).

Due to the phoswich capability of this scanner, most of the DOI is corrected and the asymmetry parameter and its impact the impact on the TDCC is relatively small. Nevertheless without DOI correction (joining data from Front and Back layers of detectors), the asymmetry increases considerably and significant differences in the Fourier Coefficients with respect the ideal case can be noticed (Table 5).

\section{CONCLUSIONS}

These results imply that because of the physical effects that blurs PET data, TDCC are only approximately valid. Therefore methods based on them, like automatic attenuation correction and sinogram restoration, should pay attention to the this blurring and its asymmetry.

Nevertheless, the impact on TDCC for devices with good DOI compensation like the eXplore Vista scanner is unimportant and may be masked by noise or scatter effects.

\section{PRACTICAL CONSIDERATIONS}

In order to avoid the errors introduced by the asymmetry of the blurring PSF, its main impact on the projection data can be minimized by positioning carefully the LOR in the center of mass of the probability distribution of the TOR. Note that this position, due to the asymmetry, doesn't match the line that connects the front face of the crystals nor the center of the crystals.

Another option to correct the TDCC for the asymmetry of the PSF is to shrink the sinogram in the radial direction before applying the TDCC. This way we can improve the results obtained when the first order TDCC is used.

\section{ACKNOWLEDGMENT}

J. L. Herraiz acknowledges support from UCM grant. Part of the computations of this work were done at the "High capacity cluster for physical techniques" of the Faculty for Physical Sciences of the UCM, funded in part by the UE under the FEDER program and in part by the UCM. This work has been partially funded by CD-TEAM, program CENIT, Ministerio de Industria, Spain.

\section{REFERENCES}

F. Natterer, The Mathematics of Computerized Tomography. Philadelphia: SIAM, 2001.

H. Sung-Cheng, "Image oscillation reduction and convergence acceleration for OS-EM reconstruction [PET imaging]," Nuclear Science, IEEE Transactions on, vol. 46, pp. 603-607, 1999.

H. Kudo and T. Saito, "Sinogram recovery with the method of convex projections for limited-data reconstruction in computed tomography," Journal of the Optical Society of America A, vol. 8, pp. 11481160, 1991.

K. Erlandsson, P. D. Esser, S. E. Strand, and R. L. van Heertum, "3D reconstruction for a multi-ring PET scanner by single-slice rebinning and axial deconvolution," Phys Med Biol, vol. 39, pp. 619-29, 1994.

A. Alessio, J. Caldwell, G. Chen, K. Branch, and P. Kinahan, "Attenuation-Emission Alignment in Cardiac PET/CT with Consistency Conditions," presented at IEEE Nuclear Science Symposium and Medical Imaging Conference, 2006.

J. L. Herraiz, S. Espana, J. M. Udias, J. J. Vaquero, and M. Desco, "Statistical Reconstruction Methods in PET: Resolution Limit, Noise, Edge Artifacts and considerations for the design of better scanners," presented at IEEE Nuclear Science Symposium and Medical Imaging Conference, 2005.

R. de-la-Prieta, J. A. Hernandez, E. Schiavi, and N. Malpica, "Analytical Geometric Model for Photon Coincidence Detection in 3D PET," presented at IEEE Nuclear Science Symposium and Medical Imaging Conference, 2006.

J. L. Herraiz, S. Espana, J. J. Vaquero, M. Desco, and J. M. Udias, "FIRST: Fast Iterative Reconstruction Software for (PET) tomography," Phys. Med. Biol., vol. 51, pp. 4547, 2006.

C. Lartizien, P. E. Kinahan, R. Swensson, C. Comtat, M. Lin, V. Villemagne, and R. Trebossen, "Evaluating image reconstruction methods for tumor detection in 3-dimensional whole-body PET oncology imaging," J Nucl Med, vol. 44, pp. 276-90, 2003.

M. Defrise, P. E. Kinahan, D. W. Townsend, C. Michel, M. Sibomana, and D. F. Newport, "Exact and 
approximate rebinning algorithms for 3-D PET data," IEEE Trans Med Imaging, vol. 16, pp. 145-58, 1997.

[11] D. Strul, R. B. Slates, M. Dahlbom, S. R. Cherry, and P. K. Marsden, "An improved analytical detector response function model for multilayer smalldiameter PET scanners," Physics in Medicine and Biology, pp. 979, 2003.

[12] A. Welch, W. Hallett, P. Marsden, and A. Bromiley, "Accurate attenuation correction in PET using short transmission scans and consistency information," Nuclear Science, IEEE Transactions on, vol. 50, pp. 427-432, 2003.

[13] A. Alessio, K. Sauer, and P. Kinahan, "Analytical reconstruction of deconvolved Fourier rebinned PET sinograms," Physics in Medicine and Biology, vol. 51, pp. 77, 2006.

[14] A. Bromiley, A. Welch, F. Chilcott, S. Waikar, S. McCallum, M. Dodd, S. Craib, L. Schweiger, and P. Sharp, "Attenuation correction in PET using consistency conditions and a three-dimensional template," Nuclear Science, IEEE Transactions on, vol. 48, pp. 1371-1377, 2001.

[15] S. Espana, J. L. Herraiz, J. J. Vaquero, M. Desco, and J. M. Udias, "PeneloPET: A MonteCarlo PET simulation tool based on PENELOPE," presented at IEEE Nuclear Science Symposium and Medical Imaging Conference, 2006.

[16] J. L. Prince and A. S. Willsky, "Hierarchical reconstruction using geometry and sinogram restoration," Image Processing, IEEE Transactions on, vol. 2, pp. 401-416, 1993.

[17] J. S. Karp, G. Muehllehner, and R. M. Lewitt, "Constrained Fourier space method for compensation of missing data in emission computed tomography," Medical Imaging, IEEE Transactions on, vol. 7, pp. 21-25, 1988. 